\title{
ESTRUTURAS RASAS DE GÁS EM SEDIMENTOS NO ESTUÁRIO POTENGI (NORDESTE DO BRASIL)
}

\author{
Eugênio Frazão ${ }^{1}$ e Helenice Vital ${ }^{1,2}$ \\ Recebido em 6 maio, 2005 / Aceito em 10 julho, 2006 \\ Received on May 6, 2005 / Accepted on July 10, 2006
}

\begin{abstract}
Areas with gas accumulations and gas seeps, where gas escapes from the river-bottom to the water column, have been mapped in the fill of a fluvial valley, the Potengi estuary. The various gas features have been classified into four types according to their specific seismic signatures: (1) acoustic blanket, (2) acoustic curtains, (3) acoustic columns, and (4) acoustic turbidity. At the same time, two types of gas escapes features have been distinguished: (1) acoustic plumes and (2) pockmarks. The neotectonic activity in the semigraben of the Potengi river may be one of the responsible factors for the migration of gas coming from the seismofacies located below the present river level where it is possible to recognize the connection with the level of the gas source through the acoustic column type seismic signature. It is concluded that sedimentary facies is the main factor determining whether gas accumulates or seeps, and also determines the specific type of accumulation or seep at each location.
\end{abstract}

Keywords: acoustic records, shallow gas accumulations, gas seeps, Potengi estuary.

RESUMO. Áreas com acumulações e escape de gás foram identificadas no estuário Potengi. As várias ocorrências de gás foram classificadas em quatro tipos, de acordo com suas assinaturas sísmicas específicas: (1) blankets ou coberturas acústicas, (2) cortinas acústicas, (3) colunas acústicas e (4) turbidez acústica. Ao mesmo tempo, dois tipos diferentes de escape de gás foram identificados: (1) plumas acústicas e (2) pockmarks. A atividade neotectônica na porção fluvial do sistema Potengi pode ser um dos responsáveis pela migração de gases provenientes das sismofácies localizadas abaixo do atual nível do rio, onde é possível reconhecer a conexão com o nível da fonte do gás, através da assinatura sísmica do tipo coluna acústica. Conclui-se que a fácies sedimentar é provavelmente o fator determinante da acumulação e escape de gás, como também do tipo específico de acumulação ou escape em cada local.

Palavras-chave: registros acústicos, acumulações de gás raso, escape de gás, estuário Potengi.

Universidade Federal do Rio Grande do Norte - UFRN, Cx. Postal 1639, 59072-970, Natal, RN, Brasil. Telefone: ++55 (84) 215-3727 R-13, 215-3808 R-213; Fax: ++55 (84) 215-3683 R-11 - E-mails: epfrazao@geologia.ufrn.br / helenice@geologia.ufrn.br

1 Programa de Pós-graduação em Geodinâmica e Geofísica - PPGG.

2 Departamento de Geologia / UFRN. 


\section{INTRODUÇÃo}

Desde os anos de 1950, diferentes anomalias nos registros por ecossonda e sonar de varredura lateral foram interpretadas, como sendo indicativas do escape de gás do leito do mar. A incorporação de levantamentos de perfilagem sísmica de subsuperfície forneceu evidências conclusivas das acumulações de gás dentro dos sedimentos do leito marinho e fluvial.

Schüller (1952) foi o primeiro a relatar 0 efeito máscara "masking", nos registros por ecossonda, do escape de gás dos sedimentos rasos marinhos. Ele chamou isso de efeito de bacia "Beckeneffekt".

Desde 0 primeiro relato de gases nos sedimentos marinhos (Emery \& Hoggan, 1958), diferentes autores noticiaram concentrações de metano e outros gases nos sedimentos marinhos próximo da superfície (Solheim \& Larson, 1987; Hovland \& Judd, 1988; Fader, 1991; Davis, 1992; Fader \& Buckley, 1995; Wever \& Fiedler, 1995; Karisiddaiah \& Veerayya, 1996; Figueiredo Jr. et al., 1996; Veerayya et al., 1998). Há também muitos trabalhos que consideram 0 aparecimento de gás em áreas costeiras como estuários e baías (Acosta, 1984; Hovland \& Judd, 1988; Fader, 1991; Taylor, 1992; Karisiddaiah et al., 1993; Pickrill, 1993; Hempel et al., 1994; Kelley et al., 1994; Vital \& Stattegger, 1997). Ao longo da costa do Estado do Rio Grande do Norte, foram encontradas acumulações de gás raso no canyon do rio Açu, na plataforma continental setentrional (Schwarzer et al., 2006).

Pockmarks no leito do mar foram primeiramente relatados por King \& McLean (1970) na plataforma continental ao largo da Nova Escócia, Canadá. Relatos posteriores de outras áreas ao redor do mundo demonstraram que pockmarks são mais que curiosidades ecológicas com apenas interesse acadêmico. A vazão do gás do leito do mar não só representa um processo erosional importante (Driscoll \& Uchupi, 1997) afetando assim a disposição das estruturas sobre 0 leito do mar, mas também desempenha um papel na produtividade biológica. Dando et al. (1994) estudaram os efeitos do vazamento de gás metano numa área de inframaré da Dinamarca e descobriram que a epifauna era mais abundante na zona do vazamento.

A propagação das ondas acústicas e sua atenuação em sedimentos gasosos ainda não são bem entendidas, apesar das freqüentes ocorrências de turbidez acústica - TA (blanking acústico das estruturas da subsuperfície) causada pela elevada impedância acústica entre sedimentos saturados de água e aqueles sedimentos com gás (Wilkens \& Richardson, 1998). A propagação acústica nos sedimentos, tipicamente de ondas com freqüências entre algumas centenas de hertz e algumas dezenas de $\mathrm{kHz}$, é fortemente afetada pela presença de gás, onde em geral as velocidades são menores em sedimentos com gás e maiores em sedimentos sem gás (Wilkens \& Richardson, 1998). A atenuação através de todas as freqüências aumenta significantemente até mesmo na presença de pequenas quantidades de gás (Yuan et al., 1992; Richardson, 1994; Fu et al., 1996).

Os sedimentos com acumulações de metano se distribuem largamente através dos oceanos do mundo (Fleischer et al., 2001). Eles se concentram em sedimentos lamosos, ricos em matéria orgânica das águas plataformais, costeiras e estuarinas, como no caso do estuário Potengi, localizado no litoral oriental do Rio Grande do Norte (Fig. 1).

0 objetivo deste trabalho foi mapear as ocorrências de estruturas rasas de gás observadas no estuário Potengi através de dados hidroacústicos com o perfilador de subfundo (tipo chirp), sonar de varredura lateral e com a ecossonda de feixe vertical.

\section{METODOLOGIA}

Para a caracterização das estruturas e feições rasas de gás no estuário Potengi foram utilizados dados obtidos durante levantamentos realizados pelo Laboratório de Geologia e Geofísica Marinha e Monitoramento Ambiental - GGEMMA da UFRN, entre os anos de 2002 e 2005. Nestes levantamentos foram realizados perfilagens sísmicas com o sistema X-Star 3200-XS da Edgetech operando na faixa de freqüência de $0.5-6.0 \mathrm{kHz}$ (18 e $9 \mathrm{~ms}$ ) e de $0.5-7.2 \mathrm{kHz}$ (30 ms), sonografias do fundo com 0 sonar de varredura lateral modelo 272-TD da Edgetech (operando na freqüência de $500 \mathrm{kHz}$ com 2 canais) e dados batimétricos adquiridos com ecobatímetro da Odom (operando na freqüência de $200 \mathrm{kHz}$ ), bem como coletas de sedimentos de fundo utilizando-se 0 amostrador pontual do tipo van Veen.

A aquisição sísmica com 0 sistema X-Star foi realizada utilizando-se pulsos de $0.5 \mathrm{~Hz}$ gerados numa taxa de pulsos de 0.5 disparos por segundo, em um sistema de 4 canais em linha. Os pulsos de reflexão sísmica rasa foram amplificados e registrados digitalmente. A velocidade sísmica pode variar entre 200$2000 \mathrm{~m} / \mathrm{s}$ em substratos inconsolidados saturados de água. Por isso, a velocidade do som na água ( $1500 \mathrm{~m} / \mathrm{s}$ ) foi usada tanto para a água como para os sedimentos a fim de se calcular a profundidade de penetração e a espessura das diferentes camadas.

0 processamento pós-aquisição dos dados sísmicos foi realizado utilizando-se o software Discover Sub-bottom para o perfilador sísmico modelo X-Star e o software Discover 560A 4.13V para o sonar de varredura lateral, ambos da Edgetech . 0 pro- 


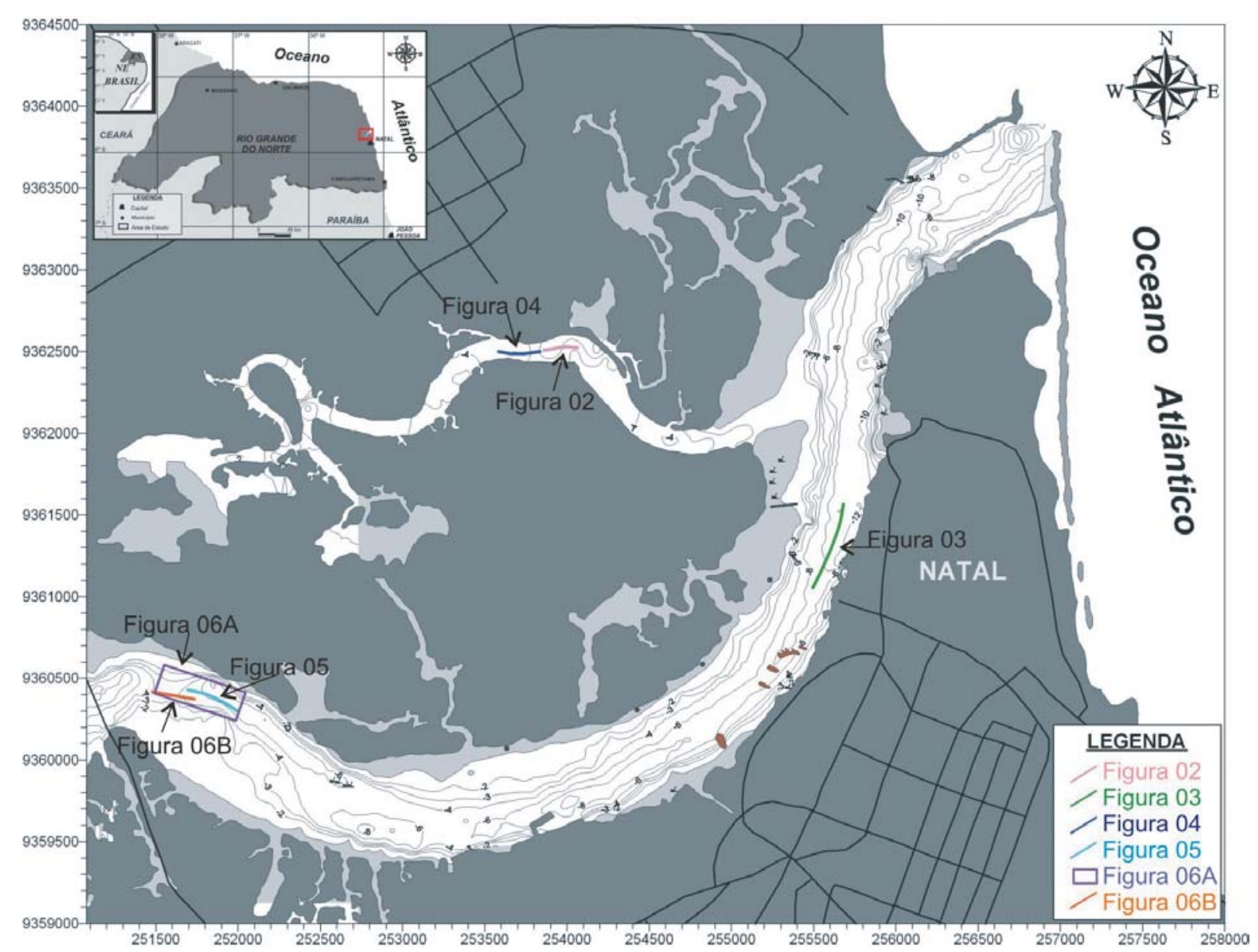

Figura 1 - Localização da área de estudo no litoral oriental do Rio Grande do Norte e mapa batimétrico simplificado, mostrando localização das Figuras 2 a 7.

cessamento consistiu basicamente no aumento do contraste, filtragem, agrupamento e a aplicação do ganho variável de tempo (TVG) para amplificar as camadas mais profundas (X-Star) e amplificar o sinal acústico da imagem adquirida (Sonar de Varredura Lateral).

As interpretações sísmicas basearam-se nos princípios da estratigrafia sísmica (Vail, 1987), e no desenvolvimento das seqüências deposicionais e partes do sistema identificando descontinuidades nas terminações dos refletores e analisando as fácies sísmicas dentro de cada seqüência sísmica (van Wagoner et al., 1987).

Para identificar os tipos de formas de leito nos sonogramas foi utilizada a classificação de Ashley (1990), incluindo as revisões de Dalrymple \& Rhodes (1995) baseadas na morfologia.

Todos os perfis foram adquiridos numa velocidade de navegação de 3.5 nós. Para o posicionamento da embarcação utilizou-se um DGPS da Furuno, com precisão de $1 \mathrm{~m}$. A profun- didade das diferentes camadas foi registrada em metros relativos ao atual nível do mar.

Ao longo do rio Potengi foram coletadas 58 amostras de sedimento de fundo distribuídas espacialmente com base em dados sonográficos e posteriormente analisadas quanto à textura segundo Folk (1974).

\section{RESULTADOS E DISCUSSÕES}

\section{Ocorrências de acumulações de gás em sedimentos}

Ao longo do estuário Potengi foram mapeadas ocorrências e acumulações de gás, utilizando-se dados hidroacústicos levando-se em conta suas assinaturas sísmicas, geometria e dimensões. A cobertura acústica ou "blankets" foi à acumulação de gás mais freqüente no estuário Potengi entre a Ponte do Igapó e sua foz (Fig. 2). Esta ocorrência de gás é identificada por uma forte reflexão superior, coerente ou interrompida (aumento da reflexão), além de mascarar completamente o registro sísmico 


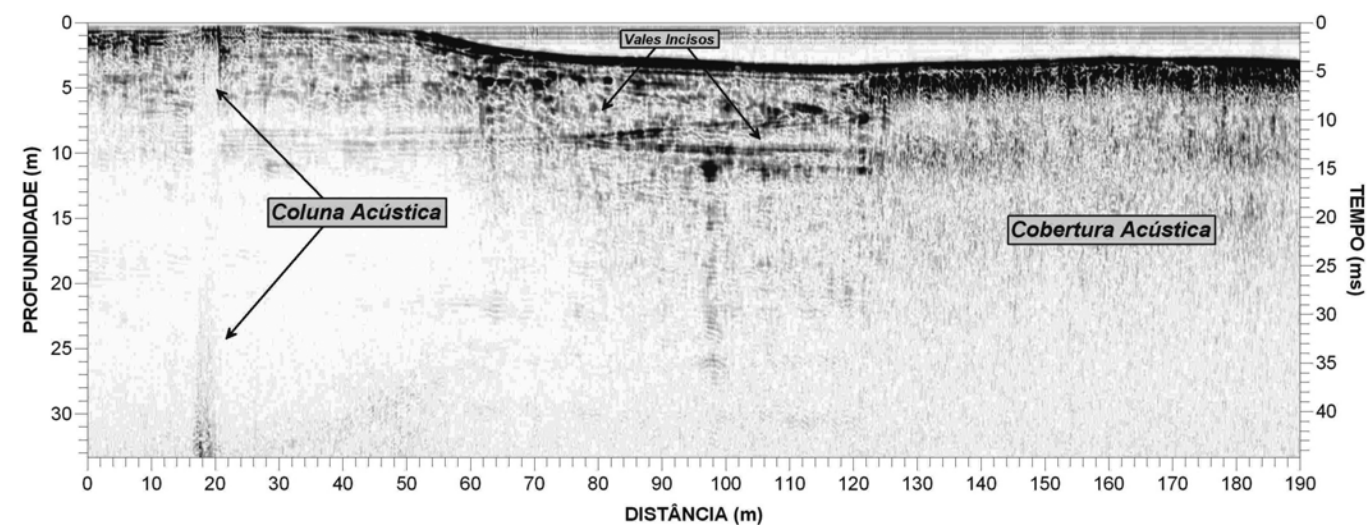

Figura 2 - Registro sísmico de alta resolução na faixa de freqüência de 0.5 a $6.0 \mathrm{kHz}$ (9ms), mostrando as ocorrências de acumulações como coluna e cobertura acústica. Para localização ver Figura 1.

subjacente sem possibilidades de estabelecer conexão com a fonte de gás.

Observa-se que na Figura 2 o limite superior deste tipo de acumulação de gás é indicado por reflexões interrompidas com uma geometria plana em $2 \mathrm{D}$ (ou suavemente inclinada). Em alguns casos (Fig. 2) há evidências de que as reflexões não são planas, mas tendem a seguir um ou dois planos de acamamento. As acumulações têm caracteristicamente 100-400 m de extensão ao longo dos perfis sísmicos com bordas muito abruptas. Esta assinatura sísmica é comparável com as coberturas acústicas descritas por Taylor (1992) e Garcia-Gil et al. (2002) e ao espaço vazio "blanking" acústico ou mascaramento "masking" acústico de Hovland \& Judd (1988).

Outras formas de ocorrência de gás mapeadas em sedimentos foram às colunas acústicas, em que são identificadas como estruturas verticais manchadas; ocasionalmente aparecem como zonas transparentes (Fig. 2). 0 limite superior apresenta uma forte reflexão com fase invertida. Neste caso, pode-se reconhecer a conexão com o nível da fonte do gás. Estas estruturas estão freqüentemente localizadas perto de acumulações de gás dos tipos "blankets" e cortinas acústicas.

Hovland \& Judd (1988) descreveram assinaturas sísmicas similares como perturbações colunares transparentes originadas pela migração ascendente dos fluídos, provavelmente gás.

$\mathrm{Na}$ Figura 3 existe a ocorrência de acumulações de gás através de cortinas acústicas ocorrendo também um completo mascaramento do registro sísmico subjacente e novamente não há a possibilidade de determinar uma conexão com a fonte de gás. Estas são características estruturais sísmicas comuns encontradas no rio Potengi (Fig. 3). Estas estruturas de gás mostram um limite superior convexo característico ou em forma de "chevron" que se apresenta como um refletor forte de fase invertida. A extensão lateral média ao longo dos perfis sísmicos atinge 20 a $50 \mathrm{~m}$. Outra característica importante das acumulações de cortinas acústicas é que a zona portadora de gás pode exibir fortes reflexões coerentes em mergulho "pull-downs" em relação aos lados da cortina devido à redução da velocidade acústica nos sedimentos portadores de gás (Fader, 1997).

Este tipo de acumulação é muito similar na forma de dimensões às cortinas de Taylor (1992) e de Garcia-Gil et al. (2002) ou ao tipo de cogumelo de Karisiddaiah et al. (1993).

A turbidez acústica foi à ocorrência de gás mais comum ao longo do estuário Potengi, que consiste de um grau variável de distúrbio no registro sísmico (Fig. 4), porém é possível seguir os refletores através do distúrbio. A origem deste tipo de acumulação é explicada pela reflexão e dispersão de energia acústica através de um grande número de bolhas de gás "in situ", provocando mascaramento nos registros sísmicos. Este efeito pode ser produzido com apenas 1\% do volume de gás no sedimento (Fannin, 1980).

\section{Ocorrência de escapes de gás}

Foi registrada evidência direta da existência de escape de gás, ou seja, bolhas de gás subindo através da coluna de água ao longo do rio Potengi. Evidência indireta resulta de vários métodos hidroacústicos como a perfilagem sísmica (operando na faixa de freqüência $0.5-7.2 \mathrm{kHz}$ ), sonar de varredura lateral (operando na freqüência de $500 \mathrm{kHz}$ ) e ecossonda vertical (operando na freqüência de $200 \mathrm{kHz}$ ), provaram ser os melhores métodos para 0 reconhecimento do escape de gás. As diferentes assinaturas acústicas da superfície sedimentar e da coluna de água identificadas foram as plumas acústicas e os pockmarks. 


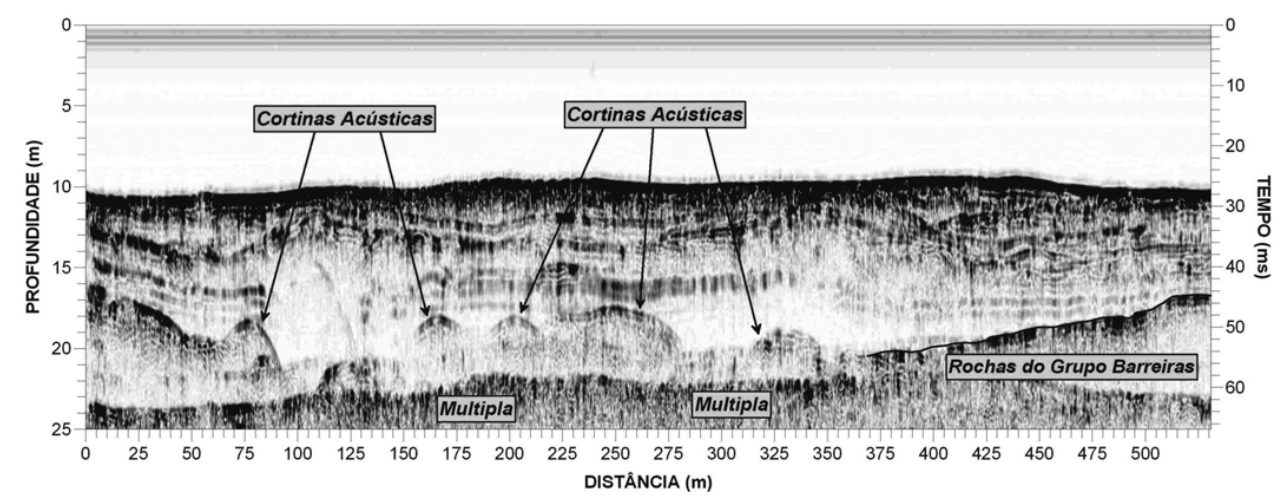

Figura 3 - Registro sísmico de alta resolução na faixa de freqüência de 0.5 a $6.0 \mathrm{kHz}$ (18ms), mostrando as ocorrências de cortinas acústicas. Para localização ver Figura 1.

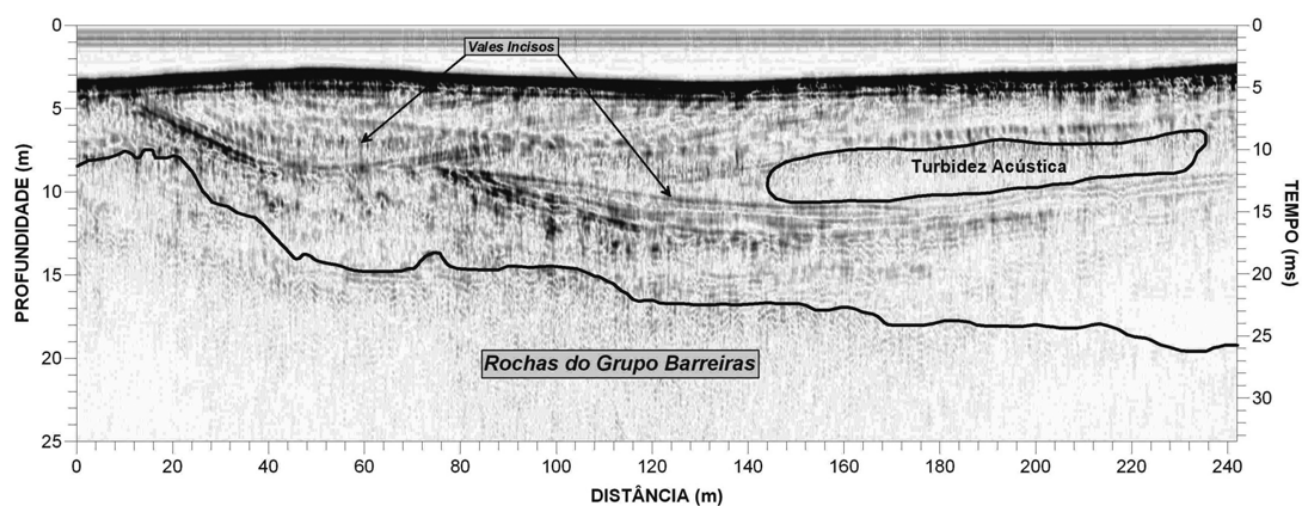

Figura 4 - Registro sísmico de alta resolução na faixa de freqüência de 0.5 a 6.0 kHz (9ms), mostrando as ocorrências de acumulações como turbidez acústica mascarando os refletores dos vales incisos. Para localização ver Figura 1.

Perfis sísmicos de alta resolução provaram ser os melhores métodos para identificação deste tipo de vazamento ao longo do estuário Potengi. Os registros acústicos mostram a presença de uma pluma de turbidez acústica subindo até $8 \mathrm{~m}$ a partir do fundo rio formando um campo de plumas (Fig. 5). Freqüentemente as plumas estão associadas à acumulação de gás (coberturas acústicas "blankets", turbidez acústica, etc.) sob o leito do rio, sendo elas a origem do gás (Fig. 5).

Taylor (1992) descreveu as plumas como uma estrutura de acumulação consistindo de uma série de reflexões parabólicas de alta amplitude com uma freqüência de ocorrência ao longo da linha sísmica de tipicamente a cada 100-200 m. Note-se que 0 autor usou o termo pluma para um tipo de acumulação de gás que não foi encontrado nesta área. Nós usamos o termo "pluma" para 0 evento de escape de gás acima descrito.

Outra ocorrência de escape de gás no estuário Potengi são os pockmarks que são considerados evidências morfológicas do escape de fluídos do leito marinho ou fluvial. Nos registros por sonar de varredura lateral os pockmarks aparecem como manchas escuras (Fig. 6a) chamados de manchas em forma de olho por Hovland (1989). No perfil batimétrico (Fig. 6b) os pockmarks apresentam estruturas em forma de "v" com 0.5-2 $\mathrm{m}$ de profundidade na superfície do leito do rio. Foi conservado o termo original "pockmark" (King \& Maclean, 1970) devido ao seu uso mundial. Hovland \& Judd (1988) confirmaram a teoria de que estas depressões se originaram devido ao escape de fluídos do leito do mar, acrescentando que, na maioria dos casos, o fluído que escapa é gás.

\section{CONCLUSÕES}

Quatro tipos diferentes de ocorrência e acumulações de gás foram identificados e caracterizados no estuário Potengi: (1) blankets ou coberturas acústicas, (2) cortinas acústicas, (3) colunas acústicas e (4) turbidez acústica. 0 mapeamento da distribuição destas estruturas possibilitou reconhecer dois importantes campos de gás no estuário Potengi: o POT-01 e o P0T-02, localiza- 


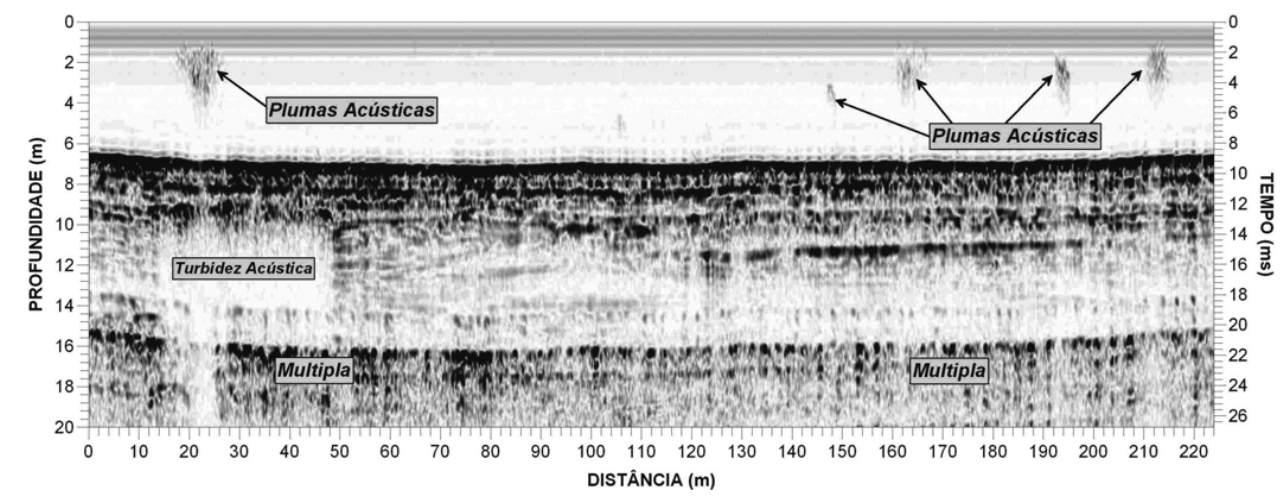

Figura 5 - Registro sísmico de alta resolução na faixa de freqüência de 0.5 a 7.2 kHz (30ms), mostrando plumas acústicas ao longo da coluna d’água, localizadas no campo de acumulações de gás P0T-01. Para localização ver Figura 1.
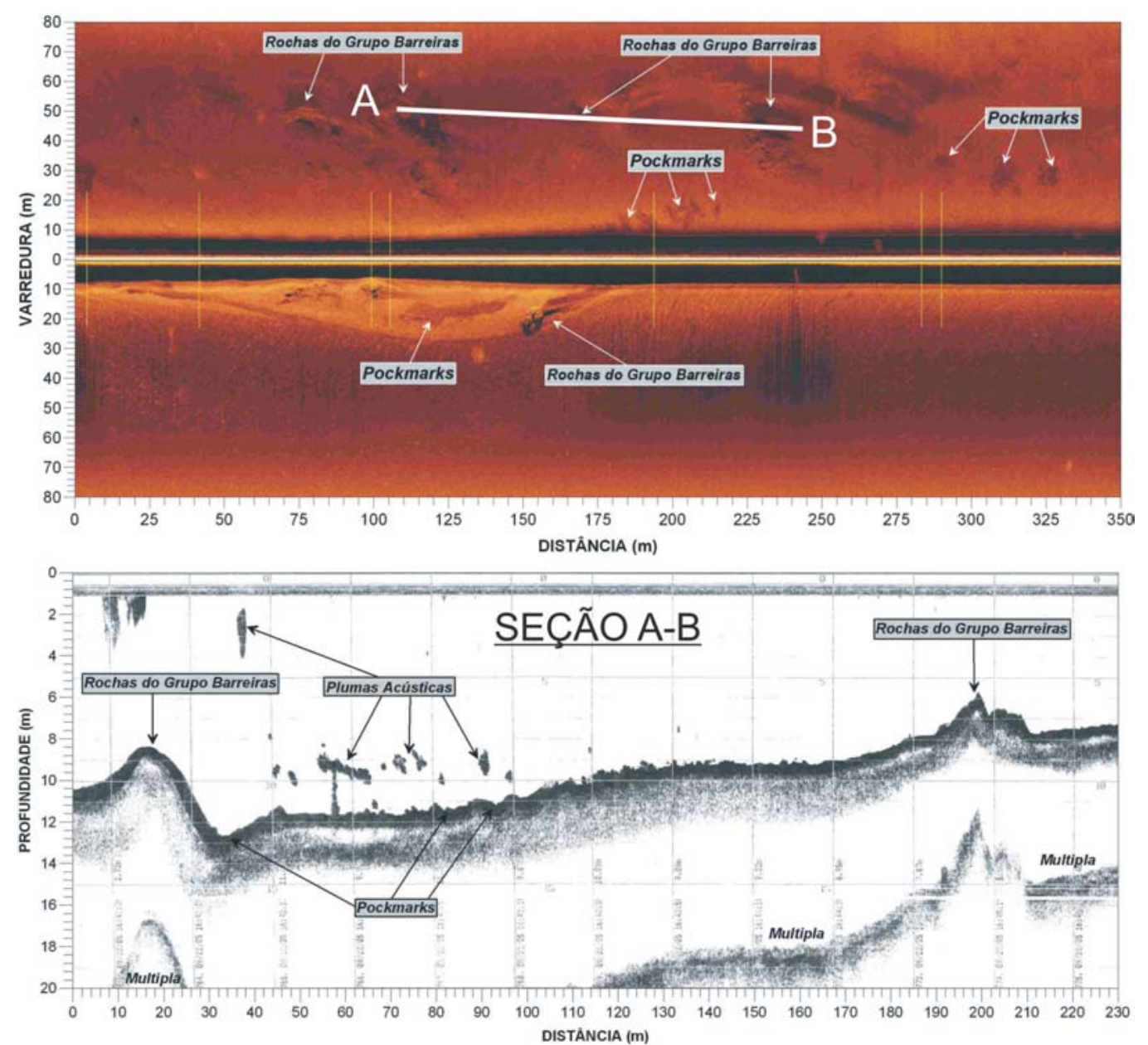

Figura 6 - Acumulações de gás no campo POT-01. (A) Registro de sonar de varredura lateral, operando na freqüência de $500 \mathrm{kHz}$, mostrando o escape de gás através dos pockmarks no campo de acumulação de gás denominado P0T-01 e (B) Perfil batimétrico adquirido na freqüência de 200 kHz, mostrando o escape de gás através dos pockmarks e plumas acústicas no campo de acumulação de gás denominado P0T-01. Para localização ver Figura 1. 


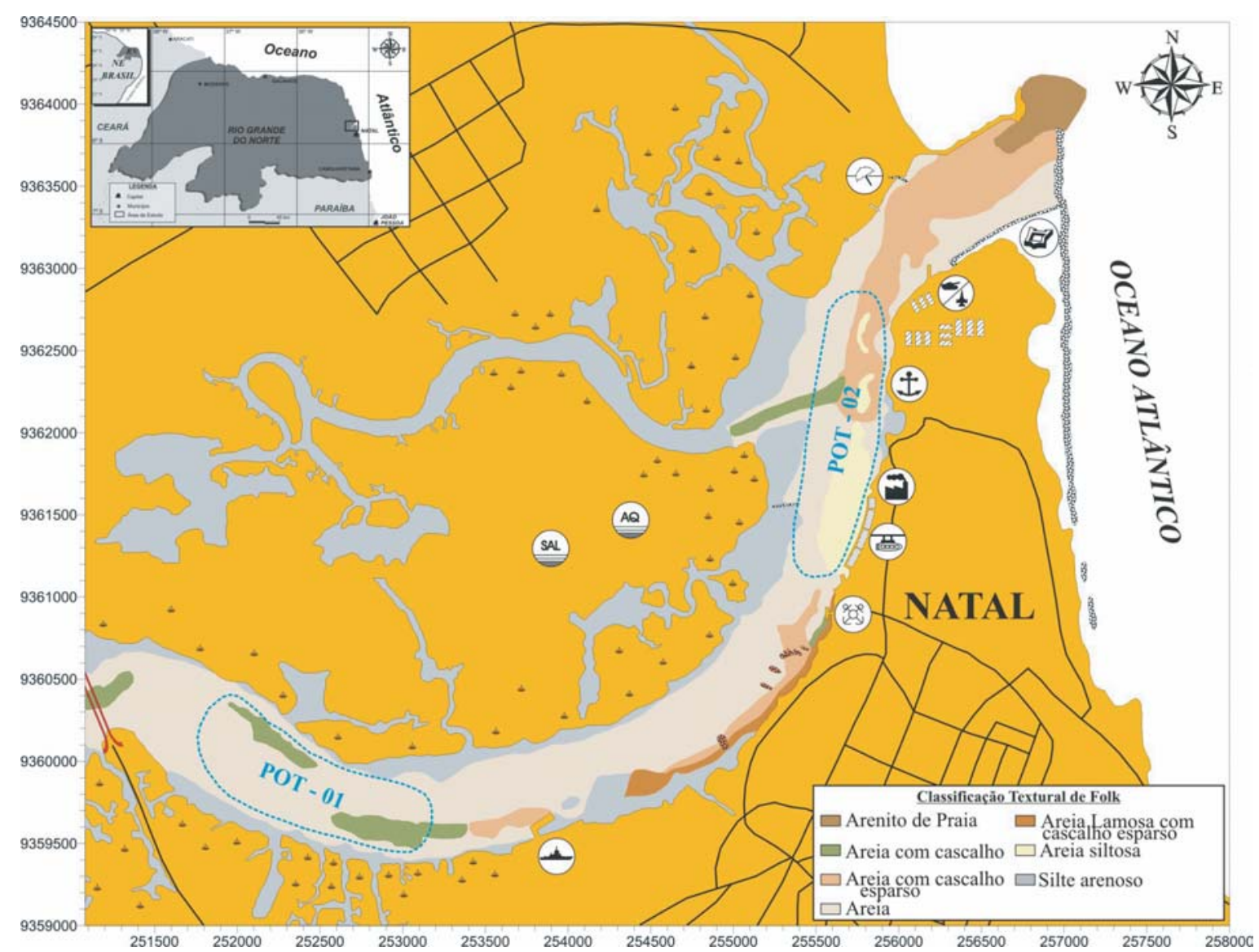

Figura 7 - Localização dos campos de acumulação gás (P0T-01 e POT-02) e mapa da distribuição superficial das principais fácies texturais do estuário Potengi, segundo a classificação de Folk (1974). Os limites de fácies foram traçados a partir da integração de dados sedimentológicos com dados sonográficos (Frazão, 2003).

dos respectivamente nas porções intermediárias e externas do rio Potengi, (Fig. 7). A correlação entre os locais das acumulações de gás e a distribuição granulométrica dos sedimentos (Fig. 7) revela uma coincidência espacial entre os campos de gás e os sedimentos superficiais finos que capeiam o rio. Estes sedimentos finos (areia siltosa) correspondem a unidades sísmicas holocênicas mais recentes (Frazão, 2003) e constitui um selante das acumulações de gás.

No campo P0T-01 existe um grande escape de gás proveniente dos sedimentos que preenchem os vales incisos. Nesta porção do estuário foi detectada a ocorrência de plumas acústicas (Figs. 5 e 6b) ao longo da coluna d'água, com a perfilagem sísmica de alta resolução e com dados batimétricos. Nesta área a porosidade das fácies suprajacentes não são eficientemente selante, já que os sedimentos superficiais que capeiam esta porção do estuário Potengi são constituídas de areia e areia com cascalho, permitindo assim a liberação do gás por diferentes tipos de escape ("pockmarks", "blankets", plumas acústicas e turbidez acústica).

Análises diretas de gás não estão disponíveis atualmente, mas a espessura limitada dos sedimentos (menos de $20 \mathrm{~m}$ ) e a profundidade rasa da água (menos de $10 \mathrm{~m}$ ) excluem a origem termogênica do gás e a presença de hidratos de gás. As pressões e temperaturas necessárias não seriam alcançadas nesta área. Assim, a degradação das bactérias da matéria orgânica, em sedimentos rasos, é considerada a mais provável causa do gás neste setor do estuário Potengi. Acosta (1984) sugeriu que 7\% da matéria orgânica são o mínimo para gerar gás suficiente para mascarar o registro sísmico. Como o metano é o único gás encontrado em quantidades significantes nos sedimentos marinhos (Floodgate \& Judd, 1992), considera-se que provavelmente o principal gás acumulado seja o metano.

0 POT-01 cobre uma área aproximada de $1.5 \mathrm{~km}^{2}$ e 0 gás se acumula em profundidade de 0 a $15 \mathrm{~m}$ abaixo do atual leito do 
rio. O POT-02 se estende por uma área com superfície em torno de $2 \mathrm{~km}^{2}$, e 0 gás parece ocorrer entre 2 a $24 \mathrm{~m}$ abaixo do leito do rio. O POT-02 (Fig. 3) ocorre, portanto no interior de horizontes mais profundos no registro sedimentar que o POT-01 (Fig. 5).

Nos dois campos, P0T-01 e P0T-02, podem ser observados zoneamentos de diferentes tipos de acumulações de gás. 0 mascaramento acústico ocorre nas partes centrais de cada campo, sendo que no POT-02 ocorre a presença de cortinas acústicas, colunas acústicas e turbidez acústica, enquanto no P0T-01 predominam acumulações de gases nos sedimentos do tipo "blankets", colunas acústicas e turbidez acústica.

A distribuição espacial dos diferentes tipos de acumulações de gás na área estudada é interpretada como evidência do controle, pelas fáceis sedimentares, porosidade e quantidade de gás dentro delas. Sugere-se que a porosidade nas fáceis onde 0 gás se acumula e as fáceis selantes são os principais controles que determinam 0 tipo de acumulação de gás. Quando há um alto contraste de porosidade entre as fáceis selantes e os sedimentos portadores de gás, então a acumulação toma a forma de "blanking" acústico. Contudo, se a porosidade das fáceis selantes é apenas um pouco mais elevada, 0 limite superior do gás será menos nítido e a acumulação seria uma cortina acústica. Independentemente da porosidade, percentagens inferiores de gás nos sedimentos causariam a formação de colunas acústicas e turbidez acústica.

\section{AGRADECIMENTOS}

Os autores agradecem a Universidade Federal do Rio Grande do Norte (UFRN) através do Programa de Pós-graduação em Geodinâmica e Geofísica (PPGG) e Laboratório de Geologia e Geofísica Marinha e Monitoramento Ambiental GGEMMA, pela infra-estrutura utilizada; a Agência Nacional do Petróleo (ANP - PRH-22) pela concessão de bolsa de pósgraduação ao primeiro autor; a Marinha do Brasil (CPRN) e ao Corpo de Bombeiros Militar do Rio Grande do Norte por disponibilizar os meios flutuantes utilizados neste estudo e aos agentes Financiadores: projetos CODERN, MARRN (FINEP-CTINFRA), PETRORISCO (REDE 05 - PETROMAR (FINEP-CTPETRO/PETROBRAS/CNPq), CNPq (Proc. No 3508811999-5 e Proc. № 500407/2004-5) e ao convênio de cooperação entre Brasil e Alemanha, envolvendo a UFRN e a Christian Albrechts Universität zu Kiel (UNIBRAL CAPES/DAAD 21-04). Agradecimentos também são devidos à equipe do GGEMMA pelo auxílio na aquisição de dados em campo.

\section{REFERÊNCIAS}

ACOSTA J. 1984. Occurrence of acoustic masking in sediments in two areas of the continental shelf of Spain: Ria de Muros (NW) and Gulf of Cádiz (SW). Marine Geology, 58: 427-434.

ASHLEY G. 1990. Classification of large-scale subaqueous bedforms a new look at an old problem. Journal of Sedimentary Petrology, 60: 160172.

DALRYMPLE RW \& RHODES RN. 1995. Estuarine dunes and bars. In: PERILLO GME (Ed.). Geomorphology and Sedimentology of Estuaries. Amsterdam Elsevier. pp. 359-422.

DANDO PR, JENSEN P, O'HARA SCM, NIVEN SJ, SCHMALJOHANN R, SCHUSTER U \& TAYLOR LJ. 1994. The effects of methane seepage at an intertidal/shallow subtidal site on the shore of the Kattegat, Vendsyssel, Denmark. Bulletin of the Geological Society of Denmark, 41: 65-79.

DAVIS AM (Ed.). 1992. Methane in marine sediments. Continental Shelf Research, 12: $175 \mathrm{pp}$.

DRISCOLL N \& UCHUPIE. 1997. The importance of gas and groundwater seepage in landscape and seascape evolution. Thalassas, 13: 355-448.

EMERY KO \& HOGGAN D. 1958. Gases in marine sediments. AAPG Bulletin, 42: 2174-2188.

FADER GBJ. 1991. Gas-related sedimentary features from the eastern Canadian continental shelf. Continental Shelf Research, 11: 1123-1153.

FADER GBJ \& BUCKLEY DE. 1995. Environmental geology of Halifax Harbour, Nova Scotia. Geoscience Canada, 22: 152-171.

FADER GBJ. 1997. The effects of shallow gas on seismic reflection profiles. In: DAVIES TA, BELL T, COOPER AK, JOSENHANS H, POLYAK L, SOLHEIM A, STOKER MS \& STRAVERS JA. (Ed.). Glaciated Continental Margins. An Atlas of Acoustic Images. Chapman \& Hall, London, pp. 29-30.

FANNIN NGT. 1980. The use of regional geological surveys in the North Sea and adjacent areas in recognition of offshore hazards. In: ARDUS DA. (Ed.). Offshore site investigation. Graham \& Trotman, London, pp. 5-21.

FIGUEIREDO Jr AG, NITTROUER CA \& COSTA EA. 1996. Gas-charged sediments in the Amazon submarine delta. Geo-Marine Letters, 16: 31-35.

FLEISCHER P, ORSI TH, RICHARDSON MD \& ANDERSON AL. 2001. Distribution of free gas in marine sediments: a global overview. GeoMarine Letters, 21: 103-122.

FLOODGATE GD \& JUDD AG. 1992. The origins of shallow gas. Continental Shelf Research, 12: 1145-1156.

FOLK RL. 1974. Petrology of Sedimentary Rocks. Austin, Texas, Hemphill Publishing Company, 181 pp. 
FRAZÃO EP. 2003. Caracterização hidrodinâmica e morfo-sedimentar do estuário Potengi e áreas adjacentes: subsídios para controle e recuperação ambiental no caso de hidrocarboneto. Dissertação de Mestrado, PPGG, UFRN, 144 pp.

FU SS, WILKENS RH \& FRAZER LN. 1996. In situ velocity profiles in gassy sediments: Kiel Bay. Geo-Marine Letters, 16: 249-253.

GARCIA-GIL S, VILAS F \& GARCIA-GARCIA A. 2002. Shallow gas features in incised-valley fills (Ría de Vigo, NW Spain): a case study. Continental Shelf Research, 22: 2303-2315.

HEMPEL P, SPIE $\beta$ V \& SCHREIBER R. 1994. Expulsion of shallow gas in the Skagerrak-evidence from sub-bottom profiling, seismic, hydroacoustical and geochemical data. Estuarine, Coastal and Shelf Science, 38: $569-582$.

HOVLAND M \& JUDD AG. 1988. Seabed Pockmarks and Seepages. Impact on Geology, Biology and the Marine Environment, Graham and Trotman, London, 294 pp.

HOVLAND M. 1989. Modern analogues to Middle Ordovician sedimentary mounds and washout depressions. Journal of Sedimentary Petrology, 59: 585-589.

KARISIDDAIAH SM, VEERAYYA M, VORA KH \& WAGLE BG. 1993. Gas-charged sediments on the inner continental shelf off western India. Marine Geology, 110: 143-152.

KARISIDDAIAH SM \& VEERAYYA M. 1996. Potential distribution of subsurface methane in the sediments of the eastern arabian sea and its possible implications. Journal of Geophysical Research, 101 (D20, D25): 887-895

KELLEY JT, DICKSON SM, BELKNAP DF, BARNHARDT WA \& HENDERSON M. 1994. Giant sea-bed pockmarks: evidence for gas escape from Belfast bay. Marine Geology, 22: 59-62.

KING LH \& McLEAN B. 1970. Pockmarks on the Scotian Shelf. Bulletin of the Geological Society of America, 81: 3141-4148.

PICKRILL RA. 1993. Shallow seismic stratigraphy and pockmarks of a hydrothermally influenced lake, Lake Rotoiti, New Zealand. Sedimentology, 40: 813-828.

RICHARDSON MD. 1994. Investigating the coastal benthic boundary layer. EOS Transactions, American Geophysical Union, 75: p. 201.

SCHÜLLER F. 1952. Untersuchungenüber die Machtigkeit von Schlickschichten mit Hilfe des Echographen. Dt. Hydrographiche Zeitung, 5: 220-231.
SCHWARZER K, STATTEGGER K, VITAL H \& BECKER M. (2006). Holocene coastal evolution of the Rio Açu Area (Rio Grande do Norte, Brazil). Journal of Coastal Research, SI 39: 141-145.

SOLHEIM A \& LARSON FR. 1987. Seismic indications of shallow gas in the Northern Barents Sea. Rapportserie Norsk Polarinstitutt no. 36, Oslo, $30 \mathrm{pp}$.

TAYLOR DI. 1992. Nearshore shallow gas around the UK coast. Continental Shelf Research, 12: 1135-1144.

VAIL PR. 1987. Seismic stratigraphy interpretation using sequence stratigraphy, part 1: Seismic stratigraphy interpretation procedure. In: BALLY AW. (Ed.). Atlas of seismic stratigraphy, vol.1, AAPG Studies in Geology, 27: $1-10$

VAN WAGONER JR, MITCHUM RM, POSAMENTIER H \& VAIL PR. 1987. Seismic stratigraphy interpretation using sequence stratigraphy, part 2: Key definitions of sequence stratigraphy. In: BALLY AW. (Ed.). Atlas of seismic stratigraphy, vol. 1, AAPG Studies in Geology, 27: 11-14.

VEERAYYA M, KARISIDDAIAH SM, VORA KH, WAGLE BG \& ALMEIDA F. 1998. Detection of gas-charged sediments and gas hydrate horizons along the western continental margin of India. In: HENRIET J-P, MIENERT J. (Ed.). Gas Hydrates. Relevance to World Margin Stability and Climate Change, Vol. 137. Special Publication. Geological Society, London, 239-253.

VITAL H \& STATTEGGER H. 1997. Gas-Charged Sediments on the Lowermost Amazon River. Anais do $5^{\circ}$ Cong. Int. Soc. Bras. Geof., São Paulo, 1: $85-88$.

WEVER TF \& FIEDLER HM. 1995. Variability of acoustic turbidity in Eckernförde Bay (Southwest Baltic Sea) related to the annual temperature cycle. Marine Geology, 125: 21-27.

WILKENS RH \& RICHARDSON MD. 1998. The influence of gas bubbles on sediment acoustic properties: in situ, laboratory, and theoretical results from Eckernförde Bay, Baltic Sea. Continental Shelf Research, 18: 1859-1892.

YUAN F, BENNELL JD \& DAVIS AM. 1992. Acoustic and physical characteristics of gassy sediments in the western Irish Sea. Continental Shelf Research, 12: 1121-1134. 


\section{NOTAS SOBRE OS AUTORES}

Eugênio Pires Frazão. Bacharel em Geologia pela Universidade Federal do Pará - UFPA e Mestre em Geodinâmica e Geofísica pela Universidade Federal do Rio Grande do Norte - UFRN, onde encontra-se atualmente desenvolvendo Tese de Doutorado na área de Geologia e Geofísica Marinha. Bolsista da Agência Nacional do Petróleo DSc.II: ANP-MME-PRH22.

Helenice Vital. Bacharel em Geologia pela Universidade Federal do Rio Grande do Norte - UFRN e Mestre em Geologia Ambiental e Marinha pela Universidade Federal do Pará - UFPA. Concluiu sua Tese de Doutorado na Christian Albrechts Universität zu Kiel, Alemanha em 1996 e desde então vem desenvolvendo projetos de ensino e pesquisa na área de Geologia e Geofísica Marinha e Monitoramento Ambiental na Universidade Federal do Rio Grande do Norte. É pesquisadora do CNPq desde 1999. 